\title{
CALCULATION OF AUTOCORRELATION FUNCTIONS FOR ELECTRONIC SPECTRA
}

\author{
Dominique DEHARENG \\ Département de Chimie, Université de Liège, Sart-Tilman, B-4000 Liège 1, Belgium
}

Received 29 July 1983

\begin{abstract}
The autocorrelation function $C(t)$ associated with the vibrational motion in the $\mathrm{A}^{2} \Pi_{u}$ state of $\mathrm{N}_{2}^{+}$is calculated by three different methods. The classical method is based on classical trajectory calculations and provides good results except for secondary maxima appearing in the experimental autocorrelation function and resulting from interference effects. In the semiclassical method, the wavefunction is expanded in a basis of frozen gaussian functions. The secondary maxima are reproduced and the result is quite satisfactory. The superposition procedure consists in an expansion of the wavefunction in the eigenfunctions of the A state of $\mathrm{N}_{2}^{+}$and leads to a very simple formula for the autocorrelation function $C(t)$. The secondary maxima are accounted for and the calculated function agrees very well with experimental results.
\end{abstract}

\section{Introduction}

In the case of an electronic spectrum, Heller has shown $[1,2]$ that the intensity $I(E)$ of a band profile is equal to the Fourier transform of an overlap integral between two wave packets $|\phi(0)\rangle$ and $|\phi(t)\rangle$ :

$$
I(E) / E \propto \int_{-\infty}^{\infty} \mathrm{d} t \exp (\mathrm{i} E t / \hbar)\langle\phi(0) \mid \phi(t)\rangle,
$$

where $|\phi(0)\rangle$ is the initial wave packet created at time $t=0$ on the potential-energy hypersurface of the upper electronic state by the spectroscopic transition, and $|\phi(t)\rangle$ is the wave packet at time $t$ as it propagates on that surface. As a consequence, a relationship can be established between the shape of an electronic band profile and the dynamic behaviour of the wave packet on the potential-energy hypersurface of the upper state. Therefore, from the knowledge of the intensity of an electronic band, one can obtain dynamic information $[3,4]$ :

$$
\langle\phi(0) \mid \phi(t)\rangle=\left(\int_{-\infty}^{\infty} \mathrm{d} E[I(E) / E] \exp (-\mathrm{i} E t / \hbar)\right)\left(\int_{-\infty}^{\infty} \mathrm{d} E[I(E) / E]\right)^{-1} .
$$

The autocorrelation function $C(t)$ associated with the electronic band profile $I(E)$ is defined as the absolute value of the overlap integral (2):

$$
C(t)=|\langle\phi(0) \mid \phi(t)\rangle| \text {. }
$$

This function provides an interesting piece of information on the nuclear motion in the electronic state under consideration and has already proven its usefulness [3-6].

The aim of the paper is to derive a method to calculate $C(t)$ as simply and rapidly as possible. For this reason, a numerical integration of the time-dependent Schrödinger equation in order to propagate the wave packet on the potential-energy surface of the upper state (by the laws of quantum mechanics) will not be attempted. Such calculations have been done by different authors [7]. We consider here three alternative methods:

(i) A classical trajectory calculation of $C(t)$, which is dealt with in section 2 .

0301-0104/84/\$03.00 @ Elsevier Science Publishers B.V.

(North-Holland Physics Publishing Division) 
(ii) A semiclassical treatment based on an expansion of $|\phi(0)\rangle$ in a basis of frozen gaussian functions whose centers follow classical trajectories, as suggested by Heller [8] (section 3).

(iii) An expansion of the initial wave packet $|\phi(0)\rangle$ in the set of vibrational eigenfunctions of the upper electronic state (section 4).

As an example, we have applied these three methods to the $\mathrm{A}^{2} \Pi_{\mathrm{u}}$ state of $\mathrm{N}_{2}^{+}$whose autocorrelation function has been experimentally determined and studied elsewhere [3].

\section{The classical method}

This method is based on a classical trajectory calculation. Such a study consists of five steps:

(A) Determination of the initial conditions of the trajectories.

(B) Choice of the statistical weights associated with the trajectories, i.e. choice of a distribution function.

(C) Representation of the potential-energy surface by an analytical expression or by a numerical interpolation procedure.

(D) Derivation and integration of the equations of motion.

(E) Calculation of the autocorrelation function.

\section{(A) Initial conditions}

If the number of variables of the system is large, the most efficient way of obtaining fast convergence of the result is to choose the initial conditions randomly via a Monte Carlo type method [9], or to generate quasi-random sequences like Halton sequences [10] of numbers in the range $(0,1)$. In the case of the $\mathrm{N}_{2}^{+}$ ion, if rotation is disregarded, only two variables are to be initialized: the internuclear distance $R$ and its conjugate momentum $P_{R}$. The initial values $R_{i}^{\text {in }}$ are obtained by scanning an interval representing the Franck-Condon zone associated with the vibrational level $v=0$ of the $\mathrm{X}^{1} \Sigma_{\mathrm{g}}^{+}$state of the molecule. This interval is $0.95 \leqslant R \leqslant 1.25 \AA$. The initial values $P_{R, i}^{\text {in }}$ are selected quasi-randomly by means of two Halton sequences, one $\left(t_{i, 1}\right)$ determining the magnitude of $P_{R, i}^{\text {in }}$, the other $\left(t_{i, 2}\right)$ its sign:

$$
P_{R, i}^{\text {in }}=\left|P_{R}^{\max }\right| t_{i, 1}(-1)^{u_{i}}, \quad i=1,2, \ldots N,
$$

where $N$ is the number of trajectories, $\left|P_{R}^{\max }\right|$ is equal to $16.62 \mathrm{au}$, corresponding to a total energy $E_{\mathrm{T}}=\left(P_{R}^{\max }\right)^{2} / 2 \mu=0.0107 \mathrm{~h}$, i.e. to a vibrational frequency for $\mathrm{N}_{2}\left(\mathrm{X}^{1} \Sigma_{\mathrm{g}}^{+}\right)$equal to $2358 \mathrm{~cm}^{-1}, \mu$ is the reduced mass of the nuclei and is equal to $7 \mathrm{a} \mathrm{Mu}=12853.05$ aum,

$$
\begin{aligned}
u_{i}=0, & \text { if } t_{i, 2}>0.5 ; \\
=1, & \text { if } t_{i, 2} \leqslant 0.5 .
\end{aligned}
$$

(B) The distribution function

It has clearly been established $[1,11,12]$ that the Wigner distribution function [13] satisfactorily simulates a distribution function of the coordinates and momenta. In the case of the $v=0$ vibrational level of the $\mathrm{X}^{1} \Sigma_{\mathrm{g}}^{+}$state of $\mathrm{N}_{2}^{+}$, this function can be written:

$$
W_{i}\left(R_{i}^{\text {in }}, P_{R, i}^{\text {in }}\right)=(\pi \hbar)^{-1} \exp \left[-2 \pi \nu \mu\left(R_{i}^{\text {in }}-R_{\mathrm{eq}_{\mathrm{x}}}\right)^{2}-\left(P_{R, i}^{\mathrm{in}}\right)^{2} / 2 \pi \nu \mu\right],
$$

where $\nu$ is the vibrational frequency of the molecule in its electronic ground state $\mathrm{X}^{1} \Sigma_{\mathrm{g}}^{+}\left(\nu=2358 \mathrm{~cm}^{-1}\right)$, $R_{\text {eq }}$ is the internuclear equilibrium distance of $\mathrm{N}_{2}$ in the $\mathrm{X}^{1} \Sigma_{\mathrm{g}}^{+}$state $\left(R_{\mathrm{eq}}=1.098 \AA\right)$ [14]. 
(C) The potential energy

The potential-energy curve is represented by the Morse function [15]

$$
V(R)=D\left\{1-\exp \left[-\beta\left(R-R_{\mathrm{eq}_{\mathrm{A}}}\right)\right]\right\}^{2},
$$

where the values of $D$ and $\beta$ have been fitted to the RKR numerical values given by Lofthus and Krupenie [14]: $D=7.75 \mathrm{eV}, \beta=2.462 \AA^{-1}$ and $R_{\mathrm{eq}_{\mathrm{A}}}=1.174 \AA$.

(D) The equations of motion

We are only interested in the autocorrelation function related to the vibrational motion of the ion. In the case of a diatomic molecule (or ion), the classical vibrational hamiltonian is:

$$
H=P_{R}^{2} / 2 \mu+V(R)=\frac{1}{2} \mu \dot{R}^{2}+V(R) .
$$

The canonical equations [16]

$$
\dot{R}=\partial H / \partial P_{R}=P_{R} / \mu, \quad \dot{P}_{R}=-\partial H / \partial R=-\partial V / \partial R
$$

are numerically integrated by the Adams-Moulton-Bashforth algorithm [17].

(E) Calculation of the autocorrelation function

Every initial value $R_{i}^{\text {in }}$ is considered as the center $R_{i}(t=0)$ of a gaussian function $\exp \left\{-a\left[R-R_{i}(t)\right]^{2}\right\}$ which evolves without distortion, i.e. with $a=$ constant. Then, the autocorrelation function is the weighted sum of the overlap integrals between initial and time-displaced gaussian functions:

$$
\begin{aligned}
C(t) & =(2 a / \pi)^{1 / 2}\left(\sum_{i=1}^{N} W_{i} \int_{-\infty}^{\infty} \mathrm{d} R \exp \left[-a\left(R-R_{i}^{\text {in }}\right)^{2}\right] \exp \left\{-a\left[R-R_{i}(t)\right]^{2}\right\}\right)\left(\sum_{j} W_{j}\right)^{-1} \\
& =\left(\sum_{i=1}^{N} W_{i} \exp \left\{-\frac{1}{2} a\left[R_{i}^{\text {in }}-R_{i}(t)\right]^{2}\right\}\right)\left(\sum_{j} W_{j}\right)^{-1} .
\end{aligned}
$$

The result depends on the value of $a$. This autocorrelation function $C(t)$ is compared with the experimental autocorrelation function obtained by Fourier transforming the intensity profile corresponding to the $\mathrm{A}^{2} \Pi_{\mathrm{u}}$ state of $\mathrm{N}_{2}^{+}$after correcting for finite experimental resolution, for rotation and for spin-orbit effects [3]. The best function $C(t)$ is obtained when $a$ is equal to the value which appears in the harmonic-oscillator wavefunction associated with the electronic ground state:

$$
\left|\Psi_{\mathrm{N}_{2}}\left(\mathrm{X}^{1} \Sigma_{\mathrm{g}}^{+}\right)\right|_{v=0}^{2} \approx(a / \pi)^{1 / 2} \exp \left[-a\left(R-R_{\mathrm{eq}_{\mathrm{X}}}\right)^{2}\right]
$$

with $a=\left(2 k_{\mathrm{X}} \mu\right)^{1 / 2} / \hbar=139.34 \mathrm{bohr}^{-2}, k_{\mathrm{X}}$ is the force constant appearing in the expansion of $V(R)$ for the $\mathrm{X}^{1} \Sigma_{\mathrm{g}}^{+}$state of $\mathrm{N}_{2}$ :

$$
V_{\mathrm{X}}(R) \approx k_{\mathrm{X}}\left(R-R_{\mathrm{eq}_{\mathrm{x}}}\right)^{2}+k_{\mathrm{XX}}\left(R-R_{\mathrm{eq}_{\mathrm{x}}}\right)^{3},
$$

$k_{\mathrm{X}}$ and $k_{\mathrm{XX}}$ are obtained from a least-squares fit to the potential energy of the $\mathrm{X}^{1} \Sigma_{\mathrm{g}}^{+}$state given by Loftus and Krupenie [14] $\left(k_{\mathrm{X}}=0.75529 \mathrm{~h} \mathrm{bohr}^{-2}, k_{\mathrm{XX}}=-0.79885 \mathrm{~h} \mathrm{bohr}^{-3}\right)$.

The graph of $C(t)$ and that of the experimental autocorrelation function are presented in fig. 1. $C(t)$ is obtained for $N=201$ classical trajectories. One sees that the agreement is rather good as far as the motion of the center of the wave packet is concerned. As a matter of fact, the shape of the recurrences and their positions are well reproduced. One observes, however, in the experimental autocorrelation function the presence of small additional peaks between the main recurrences. Their intensity increases with time. These 


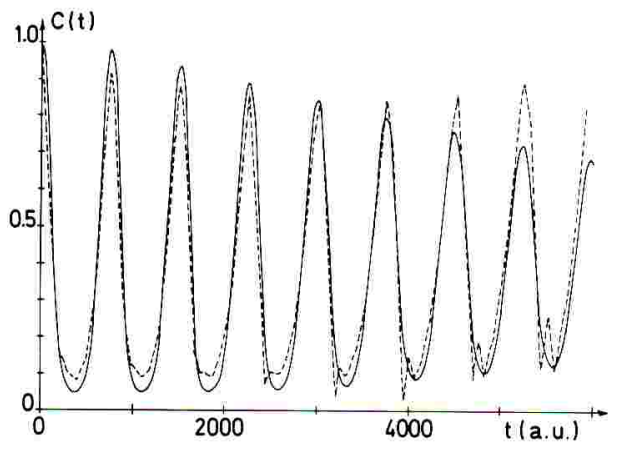

- Fig. 1. Full line: autocorrelation function $C(t)$ calculated from relation (9), where $N=201$ classical trajectories and $a=140$ bohr $^{-2}$. Dashed line: experimental autocorrelation function obtained by Fourier transformation of the intensity profile corresponding to the $\mathrm{A}^{2} \Pi_{\mathrm{u}}$ state of $\mathrm{N}_{2}^{+}$corrected for experimental finite resolution and for rotation and spin-orbit effects [3].

secondary maxima do not appear in the autocorrelation function calculated by the present method. In ref. [3], they have been interpreted as evidence for wave-packet splitting resulting from motion in an anharmonic potential. However, this interpretation seems erroneous since the present calculation is unable to account for them, in spite of the fact that the nuclear trajectories and thus the classical probability distribution have every possibility to split. As will be seen in section 4, the additional peaks are due to interference effects and cannot appear in a function calculated by a classical method.

\section{The semiclassical method}

The method used in this work has been proposed by Heller [8] and consists in the expansion of the wavefunction $\phi(R, t)$ in a basis of frozen gaussian functions $G_{j}(R, t)$ :

$$
\begin{aligned}
& \phi(R, t)=\sum_{j=1}^{N} c_{j} G_{j}(R, t), \\
& G_{j}(R, t)=(\alpha / \pi)^{1 / 4} \exp \left\{-\frac{1}{2} \alpha\left(R-R_{j, t}\right)^{2}+\mathrm{i} \hbar^{-1}\left[P_{j, t}\left(R-R_{j, t}\right)+\gamma_{j, t}\right]\right\} .
\end{aligned}
$$

They are called frozen because $\alpha$ is kept constant during the evolution of $G_{j}(R, t)$; $\alpha$ is chosen equal to the $\alpha_{\mathrm{X}}$ of eq. (26) below, i.e. $\alpha=139.34 \mathrm{bohr}^{-2}$ (see section 4).

$R_{j, t}$ and $P_{j, t}$ are the position and conjugate momentum of the center of the $j$ th gaussian function at time $t$; they obey the classical equations of motion (8). $\gamma_{j, t}$ is a phase term and has the expression

$$
\gamma_{j, t}=\int_{0}^{t}\left(P_{j, t^{\prime}}^{2} / \mu-\langle E\rangle_{j, t^{\prime}}\right) \mathrm{d} t^{\prime}
$$

where

$$
\langle E\rangle_{j, t^{\prime}}=\int_{-\infty}^{\infty} \mathrm{d} R G_{j}^{*}\left(R, t^{\prime}\right) H\left(t^{\prime}\right) G_{j}\left(R, t^{\prime}\right)
$$

and

$$
H\left(t^{\prime}\right)=\left(\hbar^{2} / 2 \mu\right) \mathrm{d}^{2} / \mathrm{d} R^{2}+V\left(R_{t^{\prime}}\right)+(\partial V / \partial R)_{R_{t^{\prime}}}\left(R-R_{t^{\prime}}\right)+\frac{1}{2}\left(\partial^{2} V / \partial R^{2}\right)_{R_{t^{\prime}}}\left(R-R_{t^{\prime}}\right)^{2} .
$$

The initial values $R_{j, 0}$ are obtained by scanning the interval $(1.053 \leqslant R \leqslant 1.143 \AA$ ) corresponding to the classical values of $R$ for the $v=0$ vibrational level of the $\mathrm{X}^{1} \Sigma_{\mathrm{g}}^{+}$state of $\mathrm{N}_{2}$ for which $E_{0}=0.00547 \mathrm{~h}$

$$
R_{j, 0}=1.053+(j / N) 0.09,
$$


where $N$ is the number of gaussian functions used in the expansion of $\phi(R, t)$ (cf. (10)). The initial values $P_{j .0}$ are chosen in such a way that all the energies corresponding to the classical trajectories $\left(R_{j, t}, P_{j, t}\right)$ are equal to $E_{0}$ :

$$
P_{j, 0}= \pm\left\{2 \mu\left[E_{0}-V\left(R_{j, 0}\right)\right]\right\}^{1 / 2} \approx \pm\left\{2 \mu\left[E_{0}-k_{\mathrm{x}}\left(R_{j, 0}-R_{\mathrm{eq}_{\mathrm{x}}}\right)^{2}\right]\right\}^{1 / 2} .
$$

The sign of $P_{j, 0}$ is chosen randomly by a Halton sequence [10] $0 \leqslant t_{j} \leqslant 1: P_{j, 0}>0$ if $t_{j}>0.5$ and $P_{j, 0}<0$ if $t_{j} \leqslant 0.5$.

For the determination of the values of the $c_{i}$ coefficients in relation (10), one has to remember that the gaussian functions as given by eq. (11) are not orthogonal. Diagonalization of the overlap matrix [18]

$$
S_{i j}=\int_{-\infty}^{\infty} G_{i}^{*}(R, 0) G_{j}(R, 0) \mathrm{d} R
$$

leads to a new basis set which is composed of orthogonal functions $F_{i}(R, 0)$ :

$$
F_{i}(R, 0)=\sum_{j=1}^{N} f_{i j} G_{j}(R, 0)
$$

with

$$
s_{i j}=\int_{-\infty}^{\infty} F_{i}^{*}(R, 0) F_{j}(R, 0) \mathrm{d} R=s_{i i} \delta_{i j} .
$$

The wavefunction $\phi(R, t)$ is expanded in this new basis set:

$$
\phi(R, t)=\sum_{i=1}^{P} g_{i} F_{i}(R, t)=\sum_{i=1}^{P} g_{i} \sum_{j=1}^{N} f_{i j} G_{i}(R, t)=\sum_{j=1}^{N}\left(\sum_{i=1}^{P} g_{i} f_{i j}\right) G_{j}(R, t) .
$$

If one compares relations (10) and (18), one sees that:

$$
c_{j}=\sum_{i=1}^{P} g_{i} f_{i j} .
$$

The $g_{i}$ can be obtained by projecting $\phi(R, 0)$ onto $F_{i}(R, 0)$ :

$$
g_{i} s_{i i}=\int_{-\infty}^{\infty} F_{i}^{*}(R, 0) \phi(R, 0) \mathrm{d} R,
$$

which becomes

$$
g_{i}=\left(\sum_{j} f_{i j}^{*} \exp \left[-\frac{1}{4} \alpha_{\mathrm{X}}\left(R_{j, 0}-R_{\mathrm{eq}}\right)^{2}-P_{j, 0}^{2} / 4 \hbar^{2} \alpha_{\mathrm{X}}+\frac{1}{2} \mathrm{i} \hbar^{-1} P_{j, 0}\left(R_{j, 0}-R_{\mathrm{eq}}\right)\right]\right)\left(s_{i i}\right)^{-1} .
$$

The autocorrelation function takes the form:

$$
\begin{aligned}
C(t)= & \mid \sum_{i=1}^{N} \sum_{j=1}^{N} c_{i}^{*} c_{j} \exp \left[-\frac{1}{4} \alpha_{\mathrm{X}}\left(R_{j, t}-R_{i, 0}\right)^{2}-\left(P_{j, t}-P_{i, 0}\right)^{2} / 4 \hbar^{2} \alpha_{\mathrm{X}}\right. \\
& \left.-\frac{1}{2} \mathrm{i} \hbar^{-1}\left(P_{j, t}+P_{i, 0}\right)\left(R_{j, t}-R_{i, 0}\right)+\mathrm{i} \hbar^{-1} \gamma_{j, t}\right] \mid .
\end{aligned}
$$

With a basis set of 15 functions, it turns out that the overlap matrix $s_{i j}$ has three diagonal elements equal 


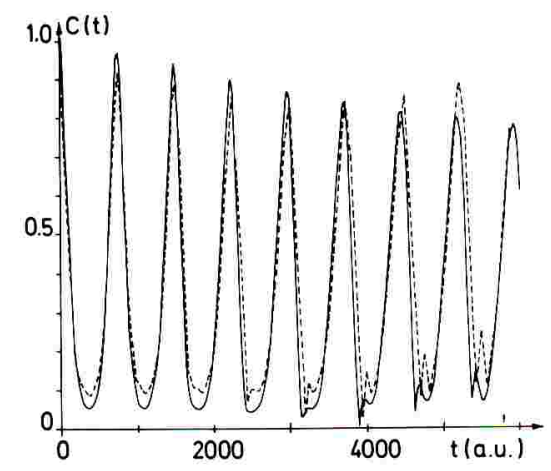

4 Fig. 2. Full line: autocorrelation function calculated by the semiclassical method (relation (22)). Dashed line: experimental autocorrelation function.

to $10^{-10}$ or less. This obviously indicates that the set contains only 12 linearly independent functions. The result is presented in fig. 2 . It is much better than the classical calculation as far as the secondary maxima are concerned. This is not surprising since the interference processes (see section 4) are taken into account by means of the phase factors $\exp \left\{\mathrm{i} \hbar^{-1}\left[P_{j, t}\left(R-R_{j, t}\right)+\gamma_{j, t}\right]\right\}$.

\section{The superposition method}

Let us expand the time-dependent wavefunction in the eigenstates of the $\mathrm{A}^{2} \Pi_{\mathrm{u}}$ state of $\mathrm{N}_{2}^{+}[19]$ :

$$
\phi(R, t)=\sum_{n} a_{n, \mathrm{~A}} \exp \left(-\mathrm{i} E_{n, \mathrm{~A}} t / \hbar\right) \zeta_{n, \mathrm{~A}}(R),
$$

where $a_{n, \mathrm{~A}}$ are the coefficients of the expansion, $E_{n, \mathrm{~A}}$ are the vibrational energies of state $\mathrm{A}$ of $\mathrm{N}_{2}^{+}$, and $\zeta_{n, \mathrm{~A}}(R)$ are the orthonormal eigenfunctions of state A. Thus, $C(t)$ can be written as follows:

$$
C(t)=|\langle\phi(0) \mid \phi(t)\rangle|=\left.\left|\sum_{n=0}^{M}\right| a_{n, \mathrm{~A}}\right|^{2} \exp \left(-\mathrm{i} E_{n, \mathrm{~A}} t / \hbar\right) \mid,
$$

where $M$ is the vibrational quantum number of the last basis function in the expansion (23) and can range from 14 to 24 without practically any change in $C(t) . a_{n . \mathrm{A}}$ is given by

$$
a_{n, \mathrm{~A}}=\int_{-\infty}^{\infty} \mathrm{d} R \zeta_{n, \mathrm{~A}}^{*}(R) \phi(R, 0) .
$$

The Franck-Condon factors $\left|a_{n, \mathrm{~A}}\right|^{2}$ and the energies $E_{n, \mathrm{~A}}$ can be either obtained from the intensity profile corresponding to state A or calculated, as done in this work.

(1) Expression of $\phi(R, 0)$

$\phi(R, 0)$ is the vibrational wavefunction of the system just after photon absorption, i.e. the vibrational wavefunction of the $v=0$ level corresponding to the $\mathrm{X}^{1} \Sigma_{\mathrm{g}}^{+}$state of the molecule. It is derived from a fourth-order perturbation expansion in the harmonic-oscillator wavefunctions

$$
\chi_{j, \mathrm{X}}(R)=N_{j, \mathrm{X}} H_{j}\left(z_{\mathrm{X}}\right) \exp \left(-\frac{1}{2} z_{\mathrm{X}}^{2}\right)
$$

where

$$
z_{\mathrm{X}}=\left(\alpha_{\mathrm{X}}\right)^{1 / 2}\left(R-R_{\mathrm{eq}}\right), \quad \alpha_{\mathrm{X}}=\left(2 k_{\mathrm{X}} \mu\right)^{1 / 2} / \hbar=139.34 \mathrm{bohr}^{-2}, \quad N_{j, \mathrm{X}}=\left(\alpha_{\mathrm{X}} / \pi\right)^{1 / 4}\left(2^{j} j !\right)^{-1 / 2}
$$

$H_{j}\left(z_{\mathrm{X}}\right)$ is the Hermite polynomial of degree $j$. 
The perturbation used is

$$
W_{\mathrm{X}}=k_{\mathrm{xx}}\left(R-R_{\mathrm{eq}_{\mathrm{x}}}\right)^{3},
$$

for which the expressions of the matrix elements are known [17]. The expansion of $\phi(R, 0)$ is made in a basis set of ten functions:

$$
\phi(R, 0)=\sum_{j=0}^{9} C_{j} \chi_{j, \mathrm{X}}(R) .
$$

(2) Calculation of $\zeta_{n, A}(R)$

The $\zeta_{n, \mathrm{~A}}(R)$ functions are the eigenfunctions of the vibrational hamiltonian $H_{\mathrm{A}}$ corresponding to state $\mathrm{A}$ of $\mathrm{N}_{2}^{+}$. The potential energy can be represented well by the Morse function (6). The $\zeta_{n, \mathrm{~A}}(R)$ functions are expanded in a basis of harmonic-oscillator wavefunctions $\chi_{j, \mathrm{~A}}(R)$ corresponding to state $\mathrm{A}$, similar to that defined in eq. (26), but with

$$
\alpha_{\mathrm{A}}=\beta(2 \mu D)^{1 / 2} / \hbar, \quad \zeta_{n, \mathrm{~A}}(R)=\sum_{j=0}^{J} b_{n, j} \chi_{j, \mathrm{~A}}(R) .
$$

$J$ can range from 29 to 49 without significant changes in $C(t)$. The $b_{n, j}$ coefficients and energies $E_{n, \mathrm{~A}}$ are obtained from the diagonalization of the matrix corresponding to the $H_{\mathrm{A}}$ operator.

The graph of $C(t)$, compared with that of the experimental autocorrelation function, is presented in fig. 3. One sees that the result is very good and accounts for the secondary maxima. Since the latter do not appear in the classical calculation, they cannot be thought of as measuring the overlap between two classical probability distributions. They are present only in treatments which involve consideration of a wavefunction, i.e. of an amplitude (see also section 3). This indicates that the appearance of subsidiary maxima is basically an interference process which, in the case of state $\mathrm{A}$ of $\mathrm{N}_{2}^{+}$is due to anharmonicity. This can be seen as follows. A wave packet moving in an anharmonic potential may break up. The wave packet should be thought of as split in the complex plane, i.e. the amplitude must be considered with its sign and its imaginary part and not as a probability distribution with several maxima on the real axis.

The following equation provides further insight into the phenomenon. Let us expand relation (24):

$$
C(t)=\left(\sum_{n=0}^{M}\left|a_{n, \mathrm{~A}}\right|^{4}+2 \sum_{n=1}^{M} \sum_{m=0}^{n-1}\left|a_{n, \mathrm{~A}}\right|^{2}\left|a_{m, \mathrm{~A}}\right|^{2} \cos \left|\left(E_{n, \mathrm{~A}}-E_{m, \mathrm{~A}}\right) t / \hbar\right|\right)^{1 / 2} .
$$

In the case of a harmonic oscillator, one can define a frequency $\omega=\hbar^{-1}\left(E_{n, \mathrm{~A}}-E_{n-1, \mathrm{~A}}\right)$; eq. (30a) then

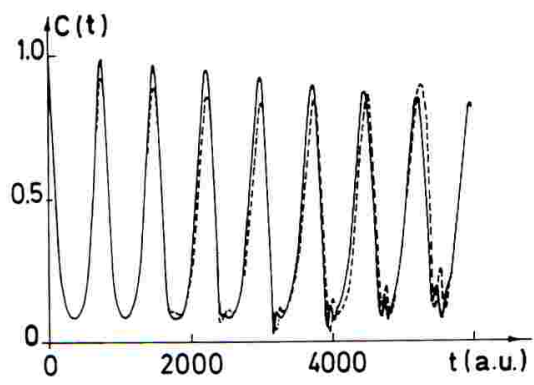

4 Fig. 3. Full line: autocorrelation function calculated from relation (24), i.e. by the superposition method. Dashed line: experimental autocorrelation function. 
reads:

$$
C(t)=\left(\sum_{n=0}^{M}\left|a_{n, \mathrm{~A}}\right|^{4}+2 \sum_{n=1}^{M} \sum_{m=0}^{n-1}\left|a_{n, \mathrm{~A}}\right|^{2}\left|a_{m, \mathrm{~A}}\right|^{2} \cos (k \omega t)\right)^{1 / 2},
$$

with $k=n-m$. For values of $t=2 \pi j / \omega, j$ being an integer, all the cosine functions and consequently $C(t)$ are strictly equal to unity, i.e. the gaussian wave packet has come back to its initial position and recovers its initial shape. In the anharmonic case, one cannot define a frequency $\omega$. As a result the various cosine functions do not reach their maximal values at the same time so that $C(t)$ cannot be equal to 1 any longer (except for very large values of $t$, around $1.21 \times 10^{-12} \mathrm{~s}$ for state $\mathrm{A}$ of $\mathrm{N}_{2}^{+}$[6]). The wave packet is distorted and interference processes occur giving rise to subsidiary maxima in $C(t)$.

Because of its simplicity, relation (24) has found other applications in the study of some dynamical processes (such as the interaction with a continuum in the strong-coupling limit [4]) allowing simple models to be built.

\section{Discussion}

As explained in section 4, the secondary maxima appearing in the autocorrelation function of state $\mathrm{A}$ of $\mathrm{N}_{2}^{+}$cannot be simply assigned to an overlap between two classical probability distributions. This is why they do not appear in the autocorrelation function calculated classically. Such structures are due to interference processes between the components of the wave packet split up by anharmonicity. This means that they can be accounted for only in treatments involving consideration of a wavefunction as in sections 3 and 4 . In addition, the breaking up of the wave packet leads to a lowering of its maximum amplitude. In the classical method, no breaking up takes place but simply a broadening. As a result, the peaks of the autocorrelation function flatten and have no longer maxima equal to unity. This fact is accounted for in the three methods of calculation considered here.

\section{Conclusions}

The determination of the autocorrelation function of an electronic band profile provides an important contribution to the analysis of the short-time dynamical behaviour of polyatomic ions or molecules. The three methods considered in this paper constitute rather easy ways, at least for diatomic molecules, to calculate an autocorrelation function of an electronic band profile corresponding to vibrational motion only.

The classical method is based on trajectory calculations and provides rather good agreement between the calculated and experimental autocorrelation functions if one does not take into account the appearance of secondary maxima due to interference processes. This method has the advantage that it can easily be used when more than one vibrational degree of freedom is concerned if one knows the potential-energy function.

The semiclassical method is based on the expansion of the wavefunction in a basis of frozen gaussian functions [8]. As these basis functions are not orthogonal, a diagonalization has to be carried out [18]. The result is better than the classical calculation but not as good as that obtained by the superposition method. Anyhow, the agreement between the calculated autocorrelation function and the experimental one is quite satisfactory. As a matter of fact, it is the best compromise between a simple classical trajectory calculation and a full-scale calculation of wave-packet propagation on a potential-energy surface. Furthermore, this method seems to be applicable without too much difficulty to the case of more than one degree of freedom [20]. 
The superposition method is based on the expansion of the wavefunction in the eigenfunctions [19] of the state under consideration; this leads to a very simple expression for the autocorrelation function. The obtained graph is in very good agreement with the graph of the experimental function. If the Franck-Condon factors and the vibrational energies of the state can be obtained, there is no difficulty in using this method in the case of more than one degree of freedom.

\section{Acknowledgement}

I would like to express my gratitude to Professor J.C. Lorquet for stimulating discussions and several comments that led to significant improvements in the manuscript. I wish to thank Dr. A.J. Lorquet for very helpful explanations about the calculation of the experimental autocorrelation function and I am grateful to Dr. A.J. Lorquet, Dr. J. Delwiche, Dr. M.J. Hubin-Franskin and Mr. B. Leyh for providing me with experimental autocorrelation functions of the $\mathrm{A}^{2} \Pi_{u}$ state of $\mathrm{N}_{2}^{+}$. This work has been supported by the Belgian Government (Action de Recherche Concertée) and the "Fonds de la Recherche Fondamentale Collective".

\section{References}

[1] E.J. Heller, J. Chem. Phys. 68 (1978) 2066.

[2] E.J. Heller, J. Chem. Phys. 68 (1978) 3891.

[3] A.J. Lorquet, J.C. Lorquet, J. Delwiche and M.J. Hubin-Franskin, J. Chem. Phys. 76 (1982) 4692.

[4] D. Dehareng, B. Leyh, M. Desouter-Lecomte, J.C. Lorquet, J. Delwiche and M.J. Hubin-Franskin, J. Chem. Phys., 79 (1983) 3719.

[5] H. Köppel, Chem. Phys. 77 (1983) 359.

[6] M. Bixon and J. Jortner, J. Chem. Phys. 77 (1982) 4175; J. Brickmann, J. Chem. Phys. 78 (1983) 1884.

[7] H.F. Harmuth, J. Math. Phys. 36 (1957) 269;

J. Mazur and R.J. Rubin, J. Chem. Phys. 31 (1959) 1395;

E.A. McCullough and R.E. Wyatt, J. Chem. Phys. 54 (1971) 3578;

E.J. Heller, J. Chem. Phys. 62 (1975) 1544;

K.C. Kulander, J. Chem. Phys. 69 (1978) 5064;

A. Askar and A.S. Cakmak, J. Chem. Phys. 68 (1978) 2794;

P.M. Agrawal and L.M. Raff, J. Chem. Phys. 74 (1981) 5076.

[8] E.J. Heller, J. Chem. Phys. 75 (1981) 2923.

[9] J.M. Hammersley and D.C. Handscomb, Monte Carlo methods (Wiley, New York, 1964).

[10] J.H. Halton, Num. Math. 2 (1960) 84.

[11] E.J. Heller, J. Chem. Phys. 65 (1976) 1289.

[12] F. Fiquet-Fayard, M. Sizun and S. Goursand, J. Phys. (Paris) 33 (1972) 669.

[13] E. Wigner, Phys. Rev. 40 (1932) 749.

[14] A. Lofthus and P.H. Krupenie, J. Phys. Chem. Ref. Data 6 (1977) 249.

[15] P.M. Morse, Phys. Rev. 34 (1929) 57.

[16] E.B. Wilson, J.C. Decius and P.C. Cross, Molecular vibrations (McGraw-Hill, New York, 1955).

[17] C.W. Gear, Numerical initial value problems in ordinary differential equations (Prentice-Hall, Englewood Cliffs, 1971),

[18] M.J. Davis and E.J. Heller, J. Chem. Phys. 71 (1979) 3383;

E.J. Heller, J. Chem. Phys. 65 (1976) 4999; 67 (1977) 3339.

[19] C. Cohen-Tannoudji, B. Diu and F. Laloë, Mécanique quantique (Hermann, Paris, 1977) ch. 5.

[20] E.J. Heller, J. Chem. Phys. 65 (1976) 4979;

K.C. Kulander and E.J. Heller, J. Chem. Phys. 69 (1978) 2439;

D.J. Tannor and E.J. Heller, J. Chem. Phys. 77 (1982) 202. 